\title{
COMPLETE BLOOD COUNT PARAMETERS IN ARTHRITIS
}

\author{
KC SR, ${ }^{1,4}$ Shrestha $S,{ }^{2}$ KC $G,{ }^{1}$ Gyawali $P^{3}$ Dahal $S,{ }^{2}$ Maharjan $B^{2}$
}

${ }^{1}$ Department of Pathology, ${ }^{2}$ Department of Orthopedics, ${ }^{3}$ Intern, KIST Medical College Teaching Hospital, ${ }^{4}$ Department of Pathology, Patan Academy of Health Sciences, Patan Hospital, Lalitpur, Nepal.

\begin{abstract}
Osteoarthritis, rheumatoid arthritis, and gout are frequently encountered diseases in the orthopedic clinic. CRP is done to evaluate and monitor these disease processes. This study aimed to evaluate total leukocyte count with differential count, neutrophil/lymphocyte ratio, platelet, platelet/lymphocyte ratio, mean platelet volume (MPV), platelet distribution width (PDW), and plateletcrit in patients with arthritis. This was a cross-sectional study conducted at KIST Medical College, Teaching Hospital from 15 Ashad 2075 to 14 Ashad 2076. The patient's demographic data and laboratory findings: total leukocyte count, differential count, hemoglobin, platelet count, plateletcrit, mean platelet volume, platelet distribution width, and plateletcrit. CRP, ESR, RA factor/anti-CCP, and Uric acid tests were also performed. Among 67 patients, osteoarthritis was seen in 53 (79.1\%), rheumatoid arthritis among 11(16.4\%), and Gout among 3(4.5\%). The ROC area under the curve was within acceptable limits for the neutrophil count, neutrophil/lymphocyte ratio, mean platelet volume, platelet distribution width. The sensitivity and specificity of the neutrophil count were $75 \%$ and $62 \%$ respectively (cut-off: $64.5 \%$ ). Neutrophil/Lymphocyte ratio was increased and correlated with CRP (p-value: <0.001). PDW had a sensitivity of 90\% and specificity of $83.0 \%$ (cut-off: $16.8 \mathrm{CV}$ ). For MPV, the sensitivity was $80 \%$ and specificity was $81 \%$ (cut-off: $9.1 \mathrm{fL}$ ). At 1.92 cut-off value, the sensitivity and specificity of neutrophil/lymphocyte ratio were $80.0 \%$ and $62.0 \%$ respectively. CBC parameters can provide an important clue to the treating physician, which helps to manage a patient with arthritis effectively. Neutrophil count, Neutrophil/Lymphocyte ratio, MPV, and PDW correlated with other inflammatory biomarkers and can be used to assess the patient with arthritis.
\end{abstract}

\section{KEYWORDS}

Arthritis, inflammatory markers; neutrophil/lymphocyte ratio, mean platelet volume, plateletcrit, platelet distribution width, platelet indices

\section{CORRESPONDING AUTHOR}

Dr. Shiva Raj KC, MD

Associate Professor and Chair,

Department of Pathology and Laboratory Medicine,

Patan Hospital, Lalitpur, Nepal

Email: shivarajkc074@gmail.com

Orcid ID: https://orcid.org/0000-0002-2107-5322

DOI: https://doi.org/10.3126/nmcj.v22i3.32621 


\section{INTRODUCTION}

Joint pain is one of the most common causes of the Orthopedic Out-Patient Department visit in any hospital. Among various causes of joint pain, osteoarthritis, rheumatoid arthritis, and gouty arthritis are some of the common diseases. Osteoarthritis (OA) is a degenerative disease of a joint which commonly involve larger joints like the knee joint, hip joints. Despite having several causes of OA, increasing age, obesity and lack of proper exercise remain the most common causes. ${ }^{1,2}$ Rheumatoid arthritis (RA), an autoimmune disease, involves small symmetrical joints and is a chronic disease, leading to limited function of the affected joints. ${ }^{3}$ Whereas, gouty arthritis is a systemic disease that occurs due to deposition of monosodium urate crystals in tissues. In patient with hyperuricaemia, these monosodium urate crystals get deposited in the joints which triggers inflammatory reaction. ${ }^{4}$

Various investigations are being performed to identify the activity of the disease processes. Among them, C-reactive protein (CRP), erythrocyte sedimentation rate (ESR) are the commonly requested tests along with complete blood count. With positive CRP, a different approach is considered for a patient with joint pain. CRP is an inflammatory marker synthesized in the liver. The CRP value is increased when there is inflammation in our body. It not only indicates an acuteinflammatory process but can be used to monitor the severity of disease in chronic conditions.

Total leukocyte count (TLC) is elevated in the inflammatory process. It is well known that neutrophilia is encountered in bacterial and acute inflammatory processes within the first few days. In the case of viral infection or chronic inflammatory process, lymphocytosis may prevail. Platelets are known to have a pivotal role in hemostasis. In addition to that, platelet plays a major role in inflammation and tissue repair. Platelet not only releases vasoactive amines like serotonin, histamine but also releases PDFG and other cytokines that have roles in the inflammatory process. ${ }^{5}$ The inflammatory process activates vascular endothelium which in turn activates platelets. Platelet production and its stimulation can be evaluated by measuring parameters, available in complete blood count parameters, like mean platelet volume (MPV), platelet distribution width (PDW), plateletcrit, platelet count. ${ }^{6}$

This study aimed to evaluate CBC parameters including platelet indices in patients with arthritis (OA, RA, and gout) and identify them as inflammatory biomarkers.

\section{MATERIALS AND METHODS}

This was a cross-sectional study conducted at KIST Medical College, Teaching Hospital from 15 Ashad 2075 to 14 Ashad 2076. Before the study, permission was obtained from the Institutional Review Board. Patients presenting with complaints of joint pain and with the diagnosis of OA, RA, and gout were explained regarding the on-going study. Once the consent was obtained, the patient's demographic data was collected. CBC was performed and laboratory findings like TLC, differential count, hemoglobin (Hb), neutrophil/lymphocyte (N/L) ratio, platelet count, platelet/lymphocyte $(\mathrm{P} / \mathrm{L})$ ratio, plateletcrit, MPV, and PDW. The manual differential count was performed only when felt necessary by the reporting pathologist. CRP, ESR, RA factor/anti-CCP, and uric acid tests were also performed. All the findings were recorded in the proforma.

Statistical Analysis: The data were entered into Microsoft Excel. Analysis of the data was done by using SPSS (statistical program for social science version 17). Variables were given as mean \pm SD and percentage. The student $t$-test was used to compare quantitative variables. Pearson correlation coefficient test ( $r$ test) was used to test the correlation between two quantitative variables. Means were compared among CRP positive and CRP negative groups. CRP was as the reference marker to confirm inflammatory process. CRP positive was considered as presence of significant ongoing inflammatory process and sensitivity of these parameters were analyzed accordingly. Linear regression test was used to find the relation between platelet indices and other parameters. Receiver operator characteristic (ROC) curve was used to find the cut-off value, sensitivity, and specificity of the MPV and PDW. A p-value of $<0.001$ is considered highly significant. If $p$ is $<0.05$, it is considered as significant, and if $\mathrm{p}>0.05$, it is considered to be insignificant.

\section{RESULTS}

During the study period, a total of 67 patients were included in the study. The mean age of the patients was 50.7 years with a minimum of 20 years and a maximum of 85 years. There was female preponderance with 16 male and 51 female ( $\mathrm{F}: \mathrm{M}=3.18: 1)$. The most common age group was $41-60$ years $(n=34 ; 50.7 \%)$, followed by $>60$ years $(n=18 ; 26.9 \%)$ and $20-40$ years $(n=15 ; 22.4 \%)$. Among all the study population, OA was the most frequently encountered disease $(n=53 ; 79.1 \%)$, followed by RA $(n=11$ $16.4 \%)$ and gout $(n=3 ; 4.5 \%)$. RA was diagnosed 
Table 1: Mean values of laboratory tests in various disease conditions among the study population $(n=67)$

\begin{tabular}{|c|c|c|c|}
\hline \multirow[b]{2}{*}{ Laboratory Tests } & \multicolumn{3}{|c|}{ Clinical Diagnosis } \\
\hline & $\begin{array}{l}\text { Osteoarthritis } \\
\text { (Mean } \pm \text { SD) }\end{array}$ & $\begin{array}{c}\text { Rheumatoid arthritis } \\
\text { (Mean } \pm \text { SD) }\end{array}$ & $\begin{array}{c}\text { Gout } \\
(\text { Mean } \pm \text { SD) }\end{array}$ \\
\hline $\operatorname{TLC}\left(\mathrm{mm}^{3}\right)$ & $7509 \pm 1922$ & $10327 \pm 3862$ & $8233 \pm 702$ \\
\hline Neutrophil (\%) & $62.9 \pm 10.5$ & $64.8 \pm 10.7$ & $68.3 \pm 0.6$ \\
\hline Lymphocyte (\%) & $34.8 \pm 10.6$ & $33.0 \pm 10.1$ & $30.0 \pm 1.7$ \\
\hline Platelet $\left(\mathrm{mm}^{3}\right)$ & $262566 \pm 75366$ & $288545 \pm 99563$ & $206666 \pm 112380$ \\
\hline ESR (mm/hr) & $10.7 \pm 7.8$ & $11.0 \pm 3.2$ & $16.0 \pm 8.0$ \\
\hline Platelet/Lymphocyte & $8290 \pm 3620$ & $9156 \pm 3372$ & $6915 \pm 3922$ \\
\hline Plateletcrit & $2.34 \pm 0.7$ & $2.7 \pm 1.1$ & $2.14 \pm 0053$ \\
\hline MPV (fL) & $8.9 \pm 1.4$ & $9.3 \pm 0.8$ & $11.9 \pm 4.9$ \\
\hline PDW (CV) & $16.6 \pm 2.1$ & $17.7 \pm 1.4$ & $15.5 \pm 5.2$ \\
\hline
\end{tabular}

Table 2: Confidence interval and p-value of various laboratory parameters in patients with positive CRP ( $n=29)$

\begin{tabular}{llllcc|} 
Tests & & & & \multicolumn{2}{c|}{$\begin{array}{c}\text { 95\% Confidence Interval of the } \\
\text { Difference }\end{array}$} \\
TLC & $\mathbf{t}$ & Df & p-value & Lower & Upper \\
Neutrophil & 16.59 & 19 & .000 & 7375 & 9504 \\
Lymphocyte & 34.09 & 19 & .000 & 66.0 & 74.6 \\
N/L ratio & 14.74 & 19 & .000 & 24.1 & 32.1 \\
Platelet & 6.22 & 19 & .000 & 2.0 & 4.1 \\
P/L ratio & 11.74 & 19 & .000 & 211149 & 302750 \\
PDW & 9.86 & 19 & .000 & 7852 & 12084 \\
MPV & 34.97 & 19 & .000 & 17.0 & 19.1 \\
Plateletcrit & 23.02 & 19 & .000 & 8.9 & 10.7 \\
\hline
\end{tabular}

with positive results of RA factor/ anti-CCP. Hyperuricemia was present in all three patients with gout. Anemia was observed in 16 (23.9\%) patients and all of them were female. The median values of laboratory findings are depicted in table 1 . The correlation between diagnosis and TLC was significant ( $p$-value $<0.01$ ). Whereas the correlation between diagnosis and differential count, platelet count, and ESR was not significant(p-value >0.05).

Among the study- population, the mean total count was highest in the patients with RA, whereas, neutrophilia was more frequent among the patients with gout. Similarly, high
ESR value was observed among patients with gout followed by RA. Platelet indices, such as MPV and PDW were elevated more among gouty patients followed by RA (Table 1). The correlation between diagnosis and $\mathrm{P} / \mathrm{L}$ ratio, Plateletcrit, MPV, and PDW were not significant ( $\mathrm{p}$-value $>0.05$ ).

CRP was done in all the patients and was positive in 20 (29.9\%) patients. Among all the patients, CRP was positive in $2 / 3(66.66 \%)$ of the patients with Gout, 13/53 (24.52\%) with OA, and 5/11 (45.45\%) with RA. Various laboratory parameters were analyzed in regards to CRP findings which is depicted in Tables 2 and 3. 
Table 3: Confidence interval and p-value of various laboratory parameters in patients with negative CRP $(n=38)$

\begin{tabular}{|c|c|c|c|c|c|}
\hline \multirow[t]{2}{*}{ Tests } & \multirow[t]{2}{*}{$\mathbf{T}$} & \multirow[t]{2}{*}{ Df } & \multirow{2}{*}{ p-value } & \multicolumn{2}{|c|}{$\begin{array}{l}\text { 95\% Confidence Interval of } \\
\text { the Difference }\end{array}$} \\
\hline & & & & Lower & Upper \\
\hline TLC & 20.61 & 46 & .000 & 7055 & 8582 \\
\hline Neutrophil & 44.03 & 46 & .000 & 57.8 & 63.3 \\
\hline Lymphocyte & 25.35 & 46 & .000 & 33.9 & 39.8 \\
\hline $\mathrm{N} / \mathrm{L}$ ratio & 18.65 & 46 & .000 & 1.6 & 1.9 \\
\hline Platelet & 24.73 & 46 & .000 & 245700 & 289235 \\
\hline $\mathbf{P} / \mathbf{L}$ ratio & 18.39 & 46 & .000 & 6849 & 8533 \\
\hline PDW & 55.78 & 46 & .000 & 15.5 & 16.7 \\
\hline MPV & 39.77 & 46 & .000 & 8.4 & 9.3 \\
\hline Plateletcrit & 21.83 & 46 & .000 & 2.1 & 2.5 \\
\hline
\end{tabular}

Table 4: Determination of sensitivity, specificity, and p-value of various variables among the study population

\begin{tabular}{lccccccc|}
\hline $\begin{array}{l}\text { Test Result } \\
\text { Variable(s) }\end{array}$ & Area & $\begin{array}{c}\text { Asymptotic 95\% } \\
\text { Confidence Interval } \\
\text { Lower }\end{array}$ & $\begin{array}{c}\text { Upper } \\
\text { Bound }\end{array}$ & $\begin{array}{c}\text { Cut-off } \\
\text { value }\end{array}$ & $\begin{array}{c}\text { Sensitivity } \\
\text { of the given } \\
\text { cut-off }\end{array}$ & $\begin{array}{c}\text { Specificity } \\
\text { of the given } \\
\text { cut-off }\end{array}$ & p-Value \\
TLC & 0.59 & 0.45 & 0.74 & NA & NA & NA & $>0.05$ \\
Neutrophil & 0.77 & 0.65 & 0.89 & 64.5 & 75.0 & 62.0 & $=0.001$ \\
Lymphocyte & 0.26 & 0.13 & 0.39 & NA & NA & NA & $>0.05$ \\
N/L ratio & 0.76 & 0.64 & 0.88 & 1.92 & 80.0 & 62.0 & $=0.001$ \\
Platelet & 0.44 & 0.28 & 0.60 & NA & NA & NA & $>0.05$ \\
P/L ratio & 0.64 & 0.49 & 0.79 & NA & NA & NA & $>0.05$ \\
PDW & 0.86 & 0.75 & 0.97 & 16.8 & 90.0 & 83.0 & $<0.001$ \\
MPV & 0.80 & 0.68 & 0.92 & 9.1 & 80.0 & 81.0 & $<0.001$ \\
Plateletcrit & 0.51 & 0.35 & 0.67 & NA & NA & NA & $>0.05$ \\
\hline
\end{tabular}

The mean of TLC, Neutrophil count, N/L ratio, PDW, MPV, and Plateletcrit were more among patients with positive than patients with negative CRP value. Platelet count was reversed among patients with positive CRP values (Table 2 \& 3). One way ANOVA was performed to compare mean values between CRP positive and negative samples. Signficant findings were observed for TLC and MPV only. For TLC , there was significant difference between groups $(F(2,60)=6.77, p=0.002$. Similarly, for MPV, $F$ $(2,64)$ was 4.9 with a p-value of 0.002 .
A Tukey post hoc test showed that in RA, TLC was statistically significant than in osteoarthritis $(p=0.001)$. Similarly, Tuckey post hoc showed that in gout MPV was statistically significiant than in OA $(p=0.007)$.

Correlation between CRP and Neutrophil count, Lymphocyte count, N/L ratio, PDW was very significant (p-value $<0.001$ ). and CRP correlated well with $\mathrm{P} / \mathrm{L}$ raio and MPV (p-value-<0.05). Lymphocyte and CRP showed negative correlation. 


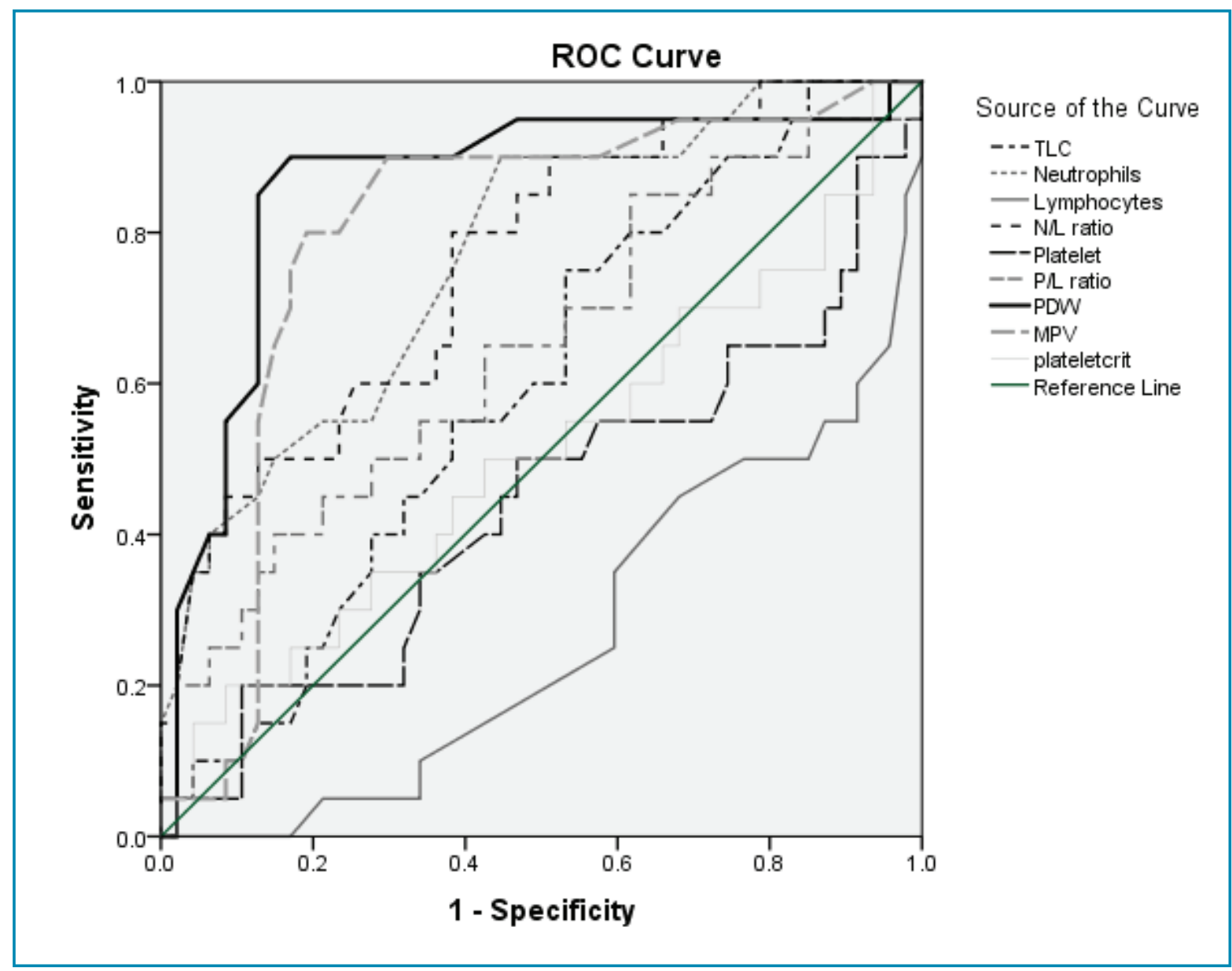

Fig. 1: ROC curve of several variables. Note that parameters such as Lymphocyte count, Platelet count, TLC, Plateletcrit had unacceptable AUC. Acceptable AUC can be seen for the Neutrophil count, N/L ratio, MPV, and PDW.

ROC curve was used to analyze the importance of platelet-derived indices along with TLC, differential count, N/L ratio, platelet count, and $\mathrm{P} / \mathrm{L}$ ratio and is shown in table 4 . The area under curve (AUC) of ROC curve of TLC, lymphocyte count, $\mathrm{P} / \mathrm{L}$ ratio, and platelet count was unacceptable $(<0.7)$ with a p-value of $>0.05$. AUC was acceptable for the neutrophil count, N/L ratio, PDW, and MPV (Table 4 and Fig.1). Among platelet-derived indices analyzed PDW had a sensitivity of $90.0 \%$ and specificity of $83.0 \%$ at the cut-off value of $16.8 \mathrm{CV}$ (Table 4). Similarly, for MPV, the sensitivity was $80.0 \%$ and specificity was $81.0 \%$ at the cut-off value of $9.1 \mathrm{fL}$.

\section{DISCUSSION}

Herein, in this study, 67 patients with arthritis who visited Orthopedic OPD were analyzed to identify the role of various $\mathrm{CBC}$ parameters that are provided by Coulter Counter. Patients with OA, RA, and gout were included in the study. We tried to analyze the correlation between the disease activity and various CBC parameters that included newer platelet indices.

C-reative protein is a well-established inflammatory marker. Other inflammatory markers such as IL-6, IL-8, procalcitonin are also an excellent inflammatory markers. ${ }^{7-9}$ But CRP is widely available tests in our context. CRP is synthesized in the liver, the increased release is seen after the secretion of IL- 6 by the macrophages and T-cells. It is an acutephase protein that binds with phospocholine and activates complement system which further promotes phagocytosis. The secretion of CRP is increased in both acute inflammatory conditions and the active phase of chronic inflammatory diseases. ${ }^{10}$

It is well-known that during acute inflammation leukocyte count increases in the peripheral blood count that slowly wander off as the inflammation subsides or transforms into chronic inflammation. In chronic inflammation, during the active phase of the disease process, TLC may increase slightly or may remain within the normal range. Furthermore, several factors 
such as sex, hormonal variations, stress level, nutrition, diet, and lifestyle may influence the TLC value. ${ }^{11}$ Because of these factors, it is difficult to decide on the activity state of chronic inflammation bases only upon TLC value. In this study, the mean TLC was more among the patient with positive CRP than patients with negative CRP value. However, AUC and p-value were not significant in regards to identifying the severity of the disease conditions.

The neutrophil is an important component of innate immunity and is elevated in several specific conditions like bacterial infections but is also elevated in nonspecific conditions like acute stress, vigorous exercise, first few hours of viral infections, and inflammations. ${ }^{12}$ The neutrophil count was higher (70 \%) in arthritis cases with positive CRP than in arthritis cases with negative CRP value (60\%). The sensitivity was $75 \%$ and specificity was $62 \%$ at the cut-off value of $64.5 \%$. Since positive CRP indicates an activephasein chronic disease, raised neutrophil value is an expected finding. Furthermore, in the active phase, as neutrophil count increases, lymphocyte count is understandably decreased. Naturally, N/L ratio should be increased. In this study, N/L ratio was increased and correlates well with CRP (p-value: <0.001). At 1.92 cutoff value, the sensitivity and specificity of $\mathrm{N} / \mathrm{L}$ ratio were $80.0 \%$ and $62.0 \%$ respectively. Uslu et al, in their study among 104 patients with RA, found N/L ratio and $\mathrm{P} / \mathrm{L}$ ratio to have aided in the assessment of disease activity. ${ }^{13}$ Similar findings were observed in another study done by Koiwa et al, who found a reduction in N/L ratio after treatment of 358 patients suffering from RA. ${ }^{14}$ In contrast to the other study, P/L ratio was not significant in this study $(p>0.05)$.

Platelet indices, such as MPV, PDW are few newer biochemical markers with promising diagnostic importance. The MPV and PDW values were more in patients with positive CRP than in patients with negative CRP. The CRP findings correlated well with MPV and PDW and were statistically significant ( $p$-value $<0.001$ ). The sensitivity and specificity of both MPV and PDW were significant at certain cut-off values. For MPV the sensitivity and specificity were $80.0 \%$ and $81.0 \%$ respectively at the cut-off value of $9.1 \mathrm{fL}$. A sensitivity of $90.0 \%$ and specificity of $83.0 \%$ was observed for PDW at the cut-off value of $16.8 \mathrm{CV}$. MPV was significantly higher in patients with RA, in a study done by Yazici et al. ${ }^{15}$ It also positively correlated with disease activity score $28 .{ }^{15}$

Although $\mathrm{OA}$ is a degenerative joint and underlying bone disease, it is associated with inflammation. In a patient with ongoing inflammation, there is increased production of proinflammatory cytokines, namely IL-6 which is released by the macrophages. This cytokine can directly stimulate the megakaryocyte, which leads to the increased rapid production of platelets. This rapid production of platelets leads to the release of large platelets along with smaller, more mature platelets. This may lead to an increase in MPV and PDW in patients with arthritis.

The cut-off value of positively correlated variables are analyzed which are neutrophil count, N/L ratio, MPV, and PDW. These tests are relatively newer and vary significantly. Hence, standardization and identification of cut-off value are required for all the inflammatory conditions, for which larger sample size, diversity of study population is necessary.

In conclusion, CBC parameters, which are readily available in the clinical laboratory, can provide an important clue to the treating physician, which helps to manage a patient with arthritis effectively. Among several parameters studied, neutrophil count, N/L ratio, MPV, and PDW correlated with other inflammatory biomarkers. Hence, with an elevated value of such parameters can be used to properly manage the patient. It would be beneficial to establish the cut-off value of these parameters that would be able to indicate the activity and severity of the inflammatory process. For this, standardization of the tests is necessary to allow its use in clinical practice.

\section{REFERENCES}

1. Litwic A, Edwards M, Dennison E, Cooper C. Epidemiology and Burden of Osteoarthritis. $\mathrm{Br}$ Med Bull 2013; 105: 185-99.

2. Ackerman IN, Osborne RH. Obesity and increased burden of hip and knee joint disease in Australia: Results from a national survey. BMC Musculoskelet Disord [Internet]. 2012; 13: 254. Available from: http://www.ncbi.nlm.nih. gov/pmc/articles/PMC3564744/

3. Guo Q, Wang Y, Xu D, Nossent J, Pavlos NJ, Xu J. Rheumatoid arthritis: pathological mechanisms and modern pharmacologic therapies. Bone Res 2018; 6: 15. doi: 10.1038/s41413-018-0016-9.

4. Ragab G, Elshahaly M, Bardin T. Gout: An old disease in new perspective - A review. J Adv Res 2017; 8: 495-511. doi: 10.1016/j.jare.2017.04.008.

5. Pokharel RK, Yadav B, Jha B, \& Parajuli K. Estimation of Serum Uric Acid in Cases of Hyperuriceamia and Gout. JNMA 2011; 51: 1520. https://doi.org/10.31729/jnma.30 
6. Ibrahim SE, Morad CS, Farouk N, Louis A. Platelet indices as markers of inflammation in systemic sclerosis patients: Relation to vascular endothelial growth factor and flow mediated dilatation. Egypt Rheumatol 2018; 40: 239-42. https://doi.org/10.1016/j.ejr.2017.12.001

7. Song J, Park DW, Moon S, Cho HJ, Park JH, Seok $\mathrm{H}$, Choi WS. Diagnostic and prognostic value of interleukin-6, pentraxin 3, and procalcitonin levels among sepsis and septic shock patients: a prospective controlled study according to the Sepsis-3 definitions. BMC Infect Dis 2019; 19: 968. doi: 10.1186/s12879-019-4618-7.

8. Shahzad A, Knapp M, Lang I, Köhler G. Interleukin 8 (IL-8) - a universal biomarker? Int'l Arch Med 2010; 3: 11. doi: 10.1186/1755-7682-311.

9. El Kassas GM, Shehata MA, El Wakeel MA, Amer AF, Elzaree FA, Darwish MK, Amer MF. Role of Procalcitonin As an Inflammatory Marker in a Sample of Egyptian Children with Simple Obesity. Open Access Maced J Med Sci 2018; 6: 1349-53. doi: 10.3889/oamjms.2018.323

10. Peppys MB, Hirschfield GM. C-reactive protein: a critical update. J Clin Invest 2003; 111: 180512. https://doi.org/10.1172/JCI18921
11. Chmielewski PP, Strzelec B. Elevated leukocyte count as a harbinger of systemic inflammation, disease progression, and poor prognosis: a review. Folia Morphol (Warsz) 2018; 77: 171-8. doi:10.5603/FM.a2017.0101

12. Kumar V, Abbas AK, Aster J. Robbins and Cotran Pathologic Basis of Disease. Philadelphia: Elsevier Saunders, 2020. 1392p.

13. Uslu AU, Küçük A, Şahin A, et al. Two new inflammatory markers associated with Disease Activity Score-28 in patients with rheumatoid arthritis: neutrophil-lymphocyte ratio and platelet-lymphocyte ratio. Int'l J Rheum Dis 2015; 18: 731-5. doi:10.1111/1756-185X.12582

14. Koiwa M, Goto S, Takahashi K, Kamada T, Takai S, Nakamura H. Neutrophil/Lymphocyte Ratio in Patients with Rheumatoid Arthritis Treated with Biological Agents. J Nippon Med Sch 2016; 83: 118-24. doi:10.1272/jnms.83.118

15. Yazici S, Yazici M, Erer B, et al. The platelet indices in patients with rheumatoid arthritis: mean platelet volume reflects disease activity. Platelets 2010; 21: 122-5. doi:10.3109/09537100903474373 